\title{
An Image Similarity Invariant Feature Extraction Method Based on Radon Transform
}

\author{
Hongjun Guo \\ Laboratory of Intelligent Information Processing, Suzhou University, Suzhou 234000, Anhui, China \\ Lili Chen* \\ Laboratory of Intelligent Information Processing, Suzhou University, Suzhou 234000, Anhui, China. \\ *Corresponding author
}

Received: August 25, 2020. Revised: March 12, 2021. Accepted: April 5, 2021. Published: April 8, 2021.

\begin{abstract}
With the advancements of computer technology, image recognition technology has been more and more widely applied and feature extraction is a core problem of image recognition. In study, image recognition classifies the processed image and identifies the category it belongs to. By selecting the feature to be extracted, it measures the necessary parameters and classifies according to the result. For better recognition, it needs to conduct structural analysis and image description of the entire image and enhance image understanding through multi-object structural relationship. The essence of Radon transform is to reconstruct the original $\mathrm{N}$-dimensional image in $\mathrm{N}$-dimensional space according to the $\mathrm{N}-1$ dimensional projection data of $\mathrm{N}$-dimensional image in different directions. The Radon transform of image is to extract the feature in the transform domain and map the image space to the parameter space. This paper study the inverse problem of Radon transform of the upper semicircular curve with compact support and continuous in the support. When the center and radius of a circular curve change in a certain range, the inversion problem is unique when the Radon transform along the upper semicircle curve is known. In order to further improve the robustness and discrimination of the features extracted, given the image translation or proportional scaling and the removal of impact caused by translation and proportion, this paper has proposed an image similarity invariant feature extraction method based on Radon transform, constructed Radon moment invariant and shown the description capacity of shape feature extraction method on shape feature by getting intra-class ratio. The experiment result has shown that the method of this paper has overcome the flaws of cracks, overlapping, fuzziness and fake edges which exist when extracting features alone, it can accurately extract the corners of the digital image and has good robustness to noise. It has effectively improved the accuracy and continuity of complex image feature extraction.
\end{abstract}

Keywords - Image similarity invariant, Feature extraction, Radon transform.

\section{INTRODUCTION}

Tn recent years, with the continuous progress of computer technology, it has become possible to simulate human visual perception task with computer, but there are still many technical difficulties, one of which is how to accurately identify the same object in different visual environments (such as view angle change, scale change, illumination change, object occlusion, etc.). The basis of target recognition is to describe the feature information of the target, so how to extract invariant features from the image is the most important. Image local feature has become the mainstream direction of image feature extraction because of its good locality, stability and invariance [1]. When studying the image, pre-process it and remove the noises to improve its signal-to-noise ratio. If the image information is too weak to be normally identified, enhancement or image transform is required prior to valid ergonomic analysis. Generally speaking, image processing includes image coding, restoration, and segmentation as well as feature extraction. Among them, feature is of critical significance and feature extraction directly affects the result of recognition and understanding. For human eyes, shape feature is an important premise to recognize an object. Therefore, the shape feature extraction and recognition technology of image has high theoretical and practical application value [2]. Conventional line feature detection methods take pixels as the unit, which differs from human visual perception system. In order to effectively analyze and process certain high-dimensional space data, image line feature extraction based on multi-scale analysis methods have witnessed rapid growth. The operation to reconstruct the image through Radon transform is called inverse Radon transform. The importance of Radon transform and inverse Radon transform on image feature detection lies in that it from the view of mathematical principle, proves that a certain fault plane of the object reconstructs the unit volume of this plane "along the projections on the attenuation distribution of straight lines", namely the possibility of 2D spatial distribution of linear attenuation distribution of voxel $[3]$. 
From the perspective of current study, shape feature extraction and description methods can be divided into contour-based method and region-based method. The contour detection method based on edge detection mainly defines the low-level abrupt changes in brightness, color and other features and it completes edge detection by marking the pixels in the image with obvious brightness variation; therefore, it is very hard to form relatively complete and closed object contour [4]. Edge detection usually convolutes image with differential operator, e.g. Sobel operator, Prewitt operator and Canny operator. This method doesn't taken into consideration the middle- and high-level visual information; so it is not at all easy to get complete and continuous contour edge and this kind of method alone is difficult to have complete object contour. Region-based shape feature extraction and recognition method utilizes all pixels in the image and contains most image information; therefore the invariant extracted can better reflect the characteristics of the image [5]. The method doesn't need to extract the boundary; instead, it directly operates on the segmented object, it has strong anti-interference capacity and it is free from boundary distortion, but as all pixels within the object in the image are considered in the recognition process, it has a cornucopia of computation. In 1917, Radon-an Australian mathematician--was the first to prove the equation of the $2 \mathrm{D}$ spatial distribution in the plane, a certain one on which the object was reconstructed through its projection. His equation requires all projections (infinite set) of all potential straight lines along this plane. The projection set obtained is termed Radon transform, which is one of the multi-scale geometric analysis methods. It implements integral with line as the unit and gets the line features of the image. This method conforms to the basic characteristics of human vision recognizing objects. At present, Radon transform has been widely applied in image detection, image restoration and many other fields [6] [7].

At first, this paper studies image feature representation, including the shape feature of cells. By analyzing its characteristics, this paper discusses the existing recognition methods, including the commonly-used image features such as color, texture, shape and spatial relationship. Then it analyzes the principle of Radon transform and its invariant moment. Base on the above research, it proposes an image similarity invariant feature extraction method based on Radon transform. Finally, the test experiment shows that the algorithm of this paper is effective.

\section{IMAge FEATURE DETECTION AND EXTRACTION}

\section{A. Features of Feature Extraction}

Depending on the basic characteristics of images, images can be divided into different categories, which are called image features. Thyroid image features include gray scale, texture, etc., which are the most basic attribute vectors used to identify an image, In general, different images have different features, and different images can be distinguished by their different attribute features. Feature extraction is used to extract such features. By extracting different types of images. The commonly used image feature extraction methods include gray feature, texture feature, shape feature and spatial relationship feature. Through the image feature extraction, the feature set which can describe the object abstractly and concretely is generated. Good feature information has geometric invariance, abstractness, limited features and cross-correlation. Each object must be represented by different features in order to distinguish it from other objects [8].

Edges are pixels that make up the edges between two image regions. Generally, the shape of an edge can be arbitrary, and it may also include intersection points. In practice, edge is generally defined as a subset of points with large gradient in the image. Some common algorithms also connect the points with high gradient to form a more perfect description of the edge. These algorithms may also put forward some restrictions on the edge. Angle is a point like feature of an image, and it has two-dimensional structure locally. Region describes the structure of a region in an image, but the region may be composed of only one pixel, so many area detection can also be used to detect the angle. In addition, the local needle has a ridge width for each ridge pixel. It is more difficult to extract ridge from gray gradient image than to extract edge, angle and region. It is used to distinguish blood vessels in images [9].

\section{B. Feature Extraction Method}

In the expression of image features, the commonly used image features are color, texture, shape and spatial relationship.

(1) Color

Color is a property parameter based on the image surface, which is a global representation of the image. It can represent the surface properties of the image area. Because it is a description of pixels, each pixel can provide effective information. Color is not sensitive and can not respond to the abrupt change of gray value. Therefore, it is impossible to describe the local features of the image, so we can only consider the global properties. The common color space includes RGB color space and HSV color space. RGB color mode is a kind of industrial color standard. It can get various colors by changing the red (R), green $(\mathrm{G})$ and blue (B) channels and their superposition. RGB represents the colors of red, green and blue channels. This standard includes almost all colors that human vision can perceive, and is one of the most widely used color systems. In HSV color space, the value range of hue $\mathrm{H}$ angle is $0^{\circ}$ to $360^{\circ}$ from red, which is $0^{\circ}$ for red, $120^{\circ}$ for green and $240^{\circ}$ for blue. Their 
complementary colors are: yellow is 60 degrees, cyan is 180 degrees, and purple is 300 degrees. Saturation S indicates how close the color is to the spectral color. A color can be seen as the result of a mixture of a spectral color and white. Histogram represents the statistical characteristics of the image region, can effectively represent the distribution of multimodal features, and it has a certain degree of rotation invariance. Various novel histogram descriptors based on different underlying features emerge in endlessly, including luminance histogram, color histogram, hog, local binary pattern histogram, etc [10].

There are four common feature extraction and matching methods: color moment, color histogram, color set and color aggregation vector.

1. Color moment

Color moment is a simple and effective color feature. Its mathematical basis is that any color distribution in an image can be represented by its moment.

In addition, because the color distribution information is mainly concentrated in the low order moments, only the first, second and third moments of color are enough to express the color distribution of images. Compared with color histogram, another advantage of this method is that it does not need to quantify the features.

First order moment:

$$
\mu_{i}=\frac{1}{N} \sum_{j=1}^{N} P_{i j}
$$

Average intensity of color components;

Second order moment:

$$
\sigma_{i}=\left[\frac{1}{N} \sum_{j=1}^{N}\left(P_{i j}-\mu_{i}\right)^{2}\right]^{\frac{1}{2}}
$$

Third order moment:

$$
s_{i}=\left[\frac{1}{N} \sum_{j=1}^{N}\left(P_{i j}-\mu_{i}\right)^{3}\right]^{\frac{1}{3}}
$$

Second and third order: variance and offset.

There are nine color moments in the image, and each color channel has three lower order moments. Color moments use only a few moments, so color moments are often used in combination with other features.

(2) Color histogram

It can simply describe the global distribution of colors in an image, that is, the proportion of different colors in the whole image. It is especially suitable for describing those images that are difficult to be automatically segmented and images that do not need to consider the spatial position of objects. But it can't describe the local distribution of colors and the spatial position of each color, that is, it can't describe a specific object or object in the image. The values in histogram are statistical, which describe the quantitative characteristics of color in the image, and can reflect the statistical distribution and basic hue of the image color.

Color histogram can be divided into three categories: global histogram, cumulative histogram and dominant color histogram.

Global histogram: it reflects the composition and distribution of colors in the image, that is, which colors appear and the probability of various colors appearing. It is not sensitive to image rotation, translation, scaling and image quality changes. It is more suitable to retrieve the global color similarity of images, that is, to measure the difference of global color distribution between two images by comparing the differences of color histograms.

Cumulative histogram: when the features in the image can not take all the available values, some zero values will appear in the statistical histogram. The appearance of these zero values will have an impact on the calculation of similarity measures, which makes the similarity measures can not correctly reflect the color difference between images. Therefore, based on the global histogram, the cumulative color histogram is used. In the cumulative histogram, adjacent colors are related in frequency. Although the amount of storage and calculation of the cumulative histogram has a small increase, the cumulative histogram eliminates the common zero value in the general histogram, and overcomes the defect that the retrieval effect of the general histogram will decline if the quantization is too fine or too coarse.

Main color histogram: because a few colors in an image often cover most of the pixels in the image, and the probability of different colors in the image is different, we can select the most frequent ones as the main color by counting the probability of various colors in the image. The use of dominant colors will not reduce the effect of color matching, because the colors in the color histogram with low frequency are not the main content of the image, to some extent, it is a kind of noise to the image content.

(3) Color set

Color set is an approximation of color histogram. Firstly, RGB color space is transformed into visual balanced color space (HSV), and the color space is quantized into several bin. Then, the image is divided into several regions by using automatic color segmentation technology. Each region is indexed by a color component of the quantized color space, so the image is expressed as a binary color index Citation table.

In image matching, the distance between different color sets and the spatial relationship of color regions are compared. Because the color set is expressed as binary feature vector, binary query tree can be constructed to speed up the retrieval, which is very powerful for large-scale image sets.

(4) Color aggregation vector

The color aggregation vector of an image is an evolution of color histogram. Its core idea is to divide the pixels belonging to each bin of the histogram into two parts: if the area of the continuous area occupied by some pixels in the bin is larger than the given threshold, the pixels in the bin are regarded as 
aggregate pixels, otherwise, they are regarded as non aggregated pixels.

The biggest feature of color aggregation vector is that it overcomes the shortcomings of color histogram and color moment, and combines the spatial information of color in image with color histogram. In this way, both the statistical information of color distribution and the spatial distribution information of color are considered.

\section{Texture}

In image texture analysis, the most important problem is to extract the feature information which can describe the texture; these features can be used to classify and describe different texture images. Texture can not represent the content of an image alone, so it is necessary to count several selected pixels within a certain range. Texture analysis refers to the process of extracting texture feature parameters through certain image processing technology, so as to obtain the quantitative or qualitative description of texture, In practice, the commonly used methods are structural method and statistical method. Roughness and directionality are the two main features that people use to distinguish texture. The element of texture feature is texture primitives. It is the most basic unit in the image. It is a set of pixels with certain shape and size, such as round spot, block spot, pattern of cloth, etc. the combination of these image primitives with certain shape and size is called texture primitives. Texture is composed of texture primitives. The density, periodicity and directionality of element arrangement are different. It can greatly change the appearance of the image [11].

The frequently-used methods to extract texture features include: gray differential statistics, gray-level co-occurrence matrix and gradient-gray level co-occurrence matrix.

(1) Gray differential statistics method

Its basic principle is to describe the gray-level changes between every pixel and the neighboring pixels of textured image. Assume that $g(x, y)$ is the pixel value at location $(x, y)$ in the image, $\Delta x$ and $\Delta y$ are the offsets along directions $x$ and $y$ respectively, then the gray-level difference between $(x, y)$ and its micro-offset point $(x+\Delta x, y+\Delta y)$ is as follows:

$$
g_{\Delta}(x, y)=g(x, y)-g(+\Delta x, y+\Delta y)
$$

Where $g_{\Delta}(x, y)$ is called the gray-level difference. Assume that all possible gray-level differential values have $m$ levels and $(x, y)$ traverses the entire image, then calculate the number of all values of $g_{\Delta}(x, y)$, get the histogram of $g_{\Delta}(x, y)$ and obtain the probability $p(i)$ of every gray-level differential value from the histogram. The following physical quantities can be defined:

$$
\text { Mean: mean }=\frac{1}{m} \sum_{i=0}^{m} i \cdot p(i)
$$

Contrast: con $=\frac{1}{m} \sum_{i=0}^{m} i^{2} \cdot p(i)$

Image entropy: ent $=-\sum_{i=0}^{m} p(i) \log _{2} p(i)$

(2) Gray-level co-occurrence matrix

Assume that $P_{x, y}$ is the gray-level co-occurrence matrix and the levels of gray-level value is $L$, the physical quantities below can be defined through gray-level co-occurrence matrix:

Energy: also known as angular second-order moment, it is the quadratic sum of the value of elements in the gray-level co-occurrence matrix and it reflects the uniformity and gray-level distribution and coarseness of texture. If all values in the matrix are the same, then the energy is small; otherwise, it is big. The calculation formula is as follows:

$$
\operatorname{asm}=\sum_{i=0}^{L-1} \sum_{j=0}^{L-1} P_{i j}^{2}
$$

Correlation: it describes the similarity between the elements of spatial gray-level co-occurrence matrix along the line and row; therefore, its value reflects the correlation of the local gray level in the image. When the element value is uniform and equal, the correlation value is big; when the element value differs greatly, it is small. The calculation formula is as below:

$$
\operatorname{cor}=\frac{1}{\sigma_{x} \sigma_{y}} \sum_{i=0}^{L-1} \sum_{j=0}^{L-1}\left(i-u_{x}\right)\left(j-u_{y}\right) P_{i j}
$$

Where

$$
\begin{gathered}
u_{x}=\frac{1}{L^{2}} \sum_{i=0}^{L-1} \sum_{j=0}^{L-1} i P_{i j} \\
u_{y}=\frac{1}{L^{2}} \sum_{i=0}^{L-1} \sum_{j=0}^{L-1} j P_{i j} \\
\sigma_{x}^{2}=\frac{1}{L^{2}} \sum_{i=0}^{L-1} \sum_{j=0}^{L-1}\left(i-u_{x}\right)^{2} P_{i j} \\
\sigma_{y}^{2}=\frac{1}{L^{2}} \sum_{i=0}^{L-1} \sum_{j=0}^{L-1}\left(j-u_{y}\right)^{2} P_{i j}
\end{gathered}
$$

Image entropy: it is the measurement for the information contained in the image and it represents the non-uniformity or complexity of textures in the image.

$$
\text { ent }=-\sum_{i=0}^{L-1} \sum_{j=0}^{L-1} P_{i j} \log _{2} P_{i j}
$$

Contrast: it is the moment of inertia near the leading diagonal in the gray-level co-occurrence matrix and it shows how the values of the matrix are distributed and reflects the clarity of 
image and the depth of texture groove. The calculation formula is as follows:

$$
\operatorname{con}=\sum_{i=0}^{L-1} \sum_{j=0}^{L-1}(i-j)^{2} P_{i j}
$$

(3) Gradient-gray level co-occurrence matrix

Gradient-gray level co-occurrence matrix (G-GLCM) extracts texture features with the general information of gray level and gradient and it takes into consideration the joint statistical distribution of gray levels of pixels and gradients of edges and its realization is similar to gray-level co-occurrence matrix. Assume that $f(x, y)$ is the pixel value of location $(x, y)$ in the image and the gray level has $L$ levels, the gradient image $g(x, y)$ of the original image can be extracted through gradient operator, and perform gray-level discretization to $g(x, y)$. Assume that the number of gray levels is $L_{g}$, then the new gray-level normalization is

$$
G(x, y)=\frac{g(x, y)-g_{\min }}{g_{\max }-g_{\min }}\left(L_{g}-1\right)
$$

The definition of gradient-gray level co-occurrence matrixis $H_{i j}=\{(x, y) \mid f(x, y)=i, G(x, y)=j\} \quad$ (then perform normalization)

\section{PRINCIPLE OF RADON TRANSFORM}

\section{A. 2D Projection}

When x-ray with an intensity of $I_{0}$ absorbs objects evenly at a rate of $\mu(x, y)$, I must reduce exponentially because of even absorption and then

$$
\begin{gathered}
I=I_{0} \exp \left\lfloor-\int_{a}^{b} \mu(y) d y\right\rfloor \\
p(\mathrm{x})=\ln \left(-\frac{I(\mathrm{x})}{I_{0}}\right)=\int_{S} \mu(\mathrm{x}, \mathrm{y}) \mathrm{dy}
\end{gathered}
$$

Where s represents the length of distance the ray travels. Assume that the slice is infinitely thin. The gray value of any point $(x, y)$ of an image is proportional to the relative linear attenuation coefficient $\mu(x, y)$ of that point [12] [13].

For any angular scanning, it needs to use rotational coordinates to describe the problem and build the rational coordinate system $(\hat{x}, \hat{y})$ above the scanning system, namely to make the bundle of rays parallel to axis $\hat{y}$ of the rotational coordinate $\operatorname{system}(\hat{x}, \hat{y})$ :
After performing rotational transform of the coordinate system, it can be obtained: $p(\hat{\mathrm{x}}, \theta)=\int_{s} \mu(\hat{\mathrm{x}}, \hat{\mathrm{y}}) d \hat{y}=\ln \left(-\frac{I(\hat{\mathrm{x}})}{I_{0}}\right)$ is the discrete value and the measured value.

The so-called projection $p(\hat{\mathrm{x}}, \theta)$ is the measured value and the integral of the absorption coefficient passing the straight line along the ray. The actual problem is to calculate the value of $\mu(x, y)$ of the integral estimated value along several straight lines. So a group of projection data can be acquired with angle $\theta$ rotating 1 degree and 180 different groups of projections can be got; in other words, after collecting the projection data of various angles $\theta$, the image of the object can be obtained, as shown in Fig.1 [14].

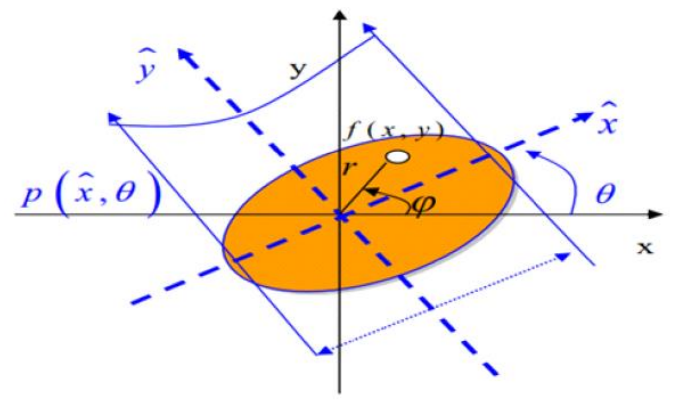

Figure 1. Data projection

\section{B. Radon Transform}

Radon transform is a transform method of calculating the projection of an image along the ray at a certain designated angle. The projection of $2 \mathrm{D}$ function $f(x, y)$ is the line integral on a determined direction, as indicated in Fig. 2 below. The line integral of 2D function $f(x, y)$ in the horizontal direction is the projection of $f(x, y)$ on axis $y$ and the linear integral of the $2 \mathrm{D}$ function is its projection on axis $x$.

Therefore, Radon transform of any angle of image $f(x, y)$ can be calculated through the projection of the function along at any angle.

$$
F_{\theta}\left(x^{\prime}\right)=\int_{-\infty}^{+\infty} f\left(x^{\prime} \cos \theta-y^{\prime} \sin \theta, x^{\prime} \cos \theta+y^{\prime} \sin \theta\right) d y^{\prime}
$$

In the equation,

$$
\left[\begin{array}{l}
x^{\prime} \\
y^{\prime}
\end{array}\right]=\left[\begin{array}{cc}
\cos \theta & \sin \theta \\
-\sin \theta & \cos \theta
\end{array}\right]\left[\begin{array}{l}
x \\
y
\end{array}\right]
$$




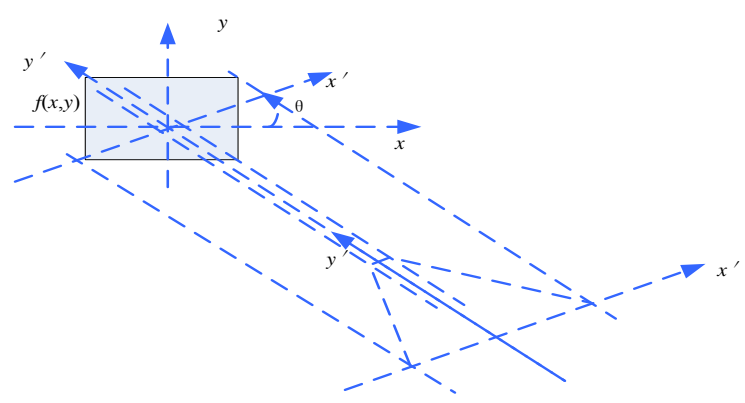

Figure 2. Projection of Radon transform

Given $b=a x+y$, map the points in the original XY plane into $\mathrm{AB}$ plane. Then all points of a straight line in the original XY plane will be situated in the same point in $\mathrm{AB}$ plane. By recording the accumulative thickness of the point in $\mathrm{AB}$ plane, it can be learnt that there exists a straight line in $\mathrm{XY}$ plane. From the accumulative peak of the corresponding point in the new plane, the significant line sets can be obtained in the original plane [15] [16].

\section{CONSTRUCTION OF INVARIANT MOMENT OF RADON TRANSFORM}

Assume that the Radon transform of the image is $p_{\theta}(t)$, then $k$ th moment can be defined as

$$
m_{k}(\theta)=\int t^{k} p_{\theta}(t) d t
$$

Obviously, $m_{0}(\theta)=m_{00} \cdot m_{00}$ is the $0^{\text {th }}$ moment of the image and it represents the image quality and it is a constant when the image is fixed. Furthermore, $\overline{t_{\theta}}=m_{1}(\theta) / m_{0}(\theta)$ represents the centroid of projection $p_{\theta}(t)$ and apparently, the common $k$ th moment doesn't have three invariants and it needs to be further constructed.

(1) Translation invariant

As image translation makes its projection move, the moment obtained also changes and translation invariance can be got by replacing the common moment with the central moment. The definition is as follows:

$$
\mu_{k}(\theta)=\int\left(t-\bar{t}_{\theta}\right)^{k} p_{\theta}(t) d t
$$

where $\overline{t_{\theta}}$ is the centroid of $p_{\theta}(t), k$ th central moment is $\left\{\mu_{k}(\theta)\right.$, and $\left.\forall \theta\right\}$ is the translation invariant; meanwhile, $\mu_{0}(\theta)=\mu_{00}=m_{00}$ is a constant and $\mu_{1}(\theta)=0, \forall \theta$.

(2) Proportion invariant

When the image changes proportionally, the Radon moment of its projection $\left(p_{\theta}^{s}(t)\right)$ also has proportional change. Assume the proportional factor is $\lambda$ and from equations (18) and (19), there is,

$$
m_{k}^{s}(\theta)=\int t^{k} p_{\theta}^{s}(t) d t=\lambda^{2+k} \int a^{k} p_{\theta}(a) d a=\lambda^{2+k} m_{k}(\theta)
$$

From above, it can be obtained that

$$
m_{0}^{s}(\theta)=\lambda^{2} m_{0}(\theta) ; m_{1}^{s}(\theta)=\lambda^{3} m_{1}(\theta)
$$

$$
\text { Make } \overline{t_{\theta}^{s}}=m_{1}^{s}(\theta) / m_{0}^{s}(\theta)=\lambda^{3} m_{1}(\theta) / \lambda^{2} m_{0}(\theta)=\overline{\lambda t_{\theta}}
$$

then the $k$ th central moment of $p_{\theta}^{s}(t)$ is

$$
\begin{aligned}
& \mu_{k}^{s}(\theta)=\int\left(t-\overline{t_{\theta}^{s}}\right)^{k} \lambda p_{\theta}\left(\frac{t}{\theta}\right) d t=\lambda \int\left(\lambda a-\overline{t_{\theta}^{s}}\right)^{k} \\
& p_{\theta}(a) \lambda d a=\lambda^{2+k} \mu_{k}(\theta)
\end{aligned}
$$

Make $\eta_{k}(\theta)=\frac{\mu_{k}(\theta)}{\mu_{0}^{j}}$

then

$\eta_{k}^{s}(\theta)=\frac{\mu_{k}^{s}(\theta)}{\left[\mu_{0}^{s}\right]^{j}}=\frac{\lambda^{2+k} \mu_{k}(\theta)}{\left[\lambda^{2} \mu_{0}\right]^{j}}=\frac{\lambda^{2+k}}{\lambda^{2 j}} \cdot \frac{\mu_{k}(\theta)}{\mu_{0}}=\frac{\lambda^{2+k}}{\lambda^{2 j}} \cdot \eta_{k}(\theta)$

Make $\frac{\lambda^{2+k}}{\lambda^{2 j}}=1$ and $\mathrm{j}=(\mathrm{k}+2) / 2$

Namely $\eta_{k}(\theta)=\frac{\mu_{k}(\theta)}{\mu_{0}^{(k+2) / 2}}$

where $\eta_{k}(\theta)$ is the proportional and translation invariant.

(3) Inverse Radon transform

With the center of the image as the origin of polar coordinates, straight line $\mathrm{X}$ is the new projection coordinate and $\theta$ is the angle.

$$
R_{J}(\theta, f)=\int_{g^{2}} f(x) \delta\left(x_{1} \cos \theta+x_{2} \sin \theta-f\right) d x
$$

As shown in equation (24), Radon transform of $f(x)$ is the projection of $f(x)$ along different $\theta$ directions and the Ridgelet transform of $f(x)$ is seen as the result of firstly performing Radon transform on $f(x)$ and then conducting $1 \mathrm{D}$ wavelet transform along every integral direction, namely

$$
C R T_{J}(a, b, \theta)=\int \psi_{a, b}(f) R_{J}(\theta, f) d t
$$

It is because Ridgelet transform performs 1D wavelet transform on every direction in Radon domain that it converts the line singularity of the image into point singularity and that it makes full use of the excellent 
representation property of wavelet transform on point singularity to get the sparse representation of the image with line singularity. Inverse Ridgelet transform can be obtained by implementing 1D inverse wavelet transform along every direction and then performing inverse Radon transform.

\section{TEST EXPERIMENT AND RESULt ANALYSIS}

To determine whether shape feature extraction is good or not is mainly measured by rotation translation and scale invariance and meanwhile by selecting the key morphological features such as cell area, kernel area, darkness of color of cell nucleus, cell perimeter, rectangularity of cell and circularity of cell. Intuitively speaking, shape can be viewed as a strong edge point and it characterizes the contour shape. The points that change rapidly along those directions of profile curve are the angular points and the points with slow change in the profile curve correspond to straight lines. Therefore, these special points play a big role in shape description and matching and they are used to compress the data to represent the important information of shape. Here, the parameters of Radon shape feature extraction are 15, 30 and 45 respectively, as shown in Fig.3 and Fig.4.

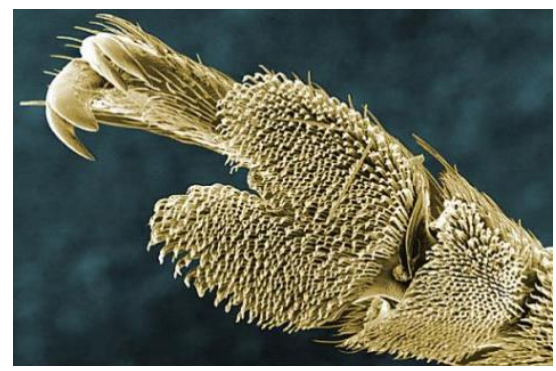

(a)Original image

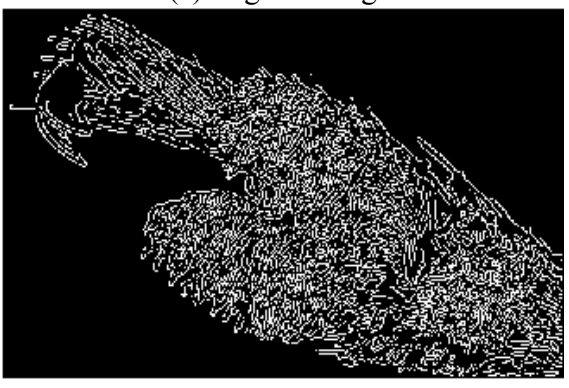

(b) Edge detection

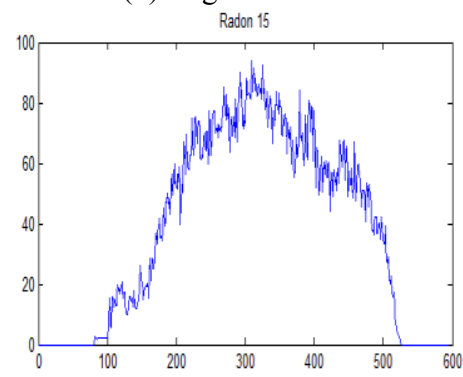

(c) Radon $=15$

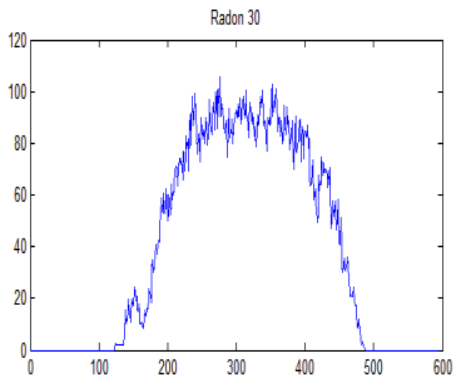

(d) Radon $=30$

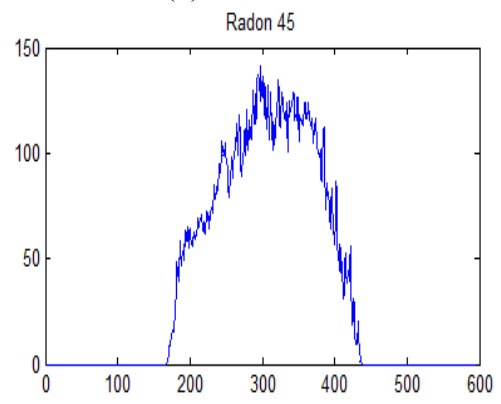

(e) $\operatorname{Radon}=45$

Figure 3. Insects 1

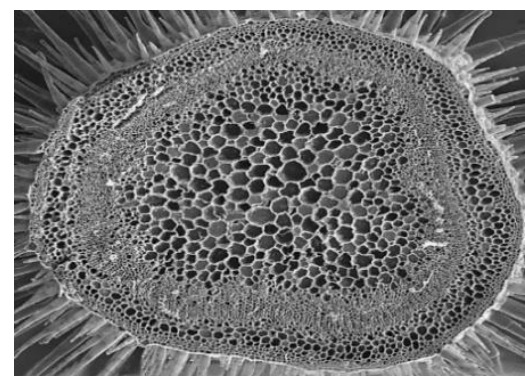

(a)Original image

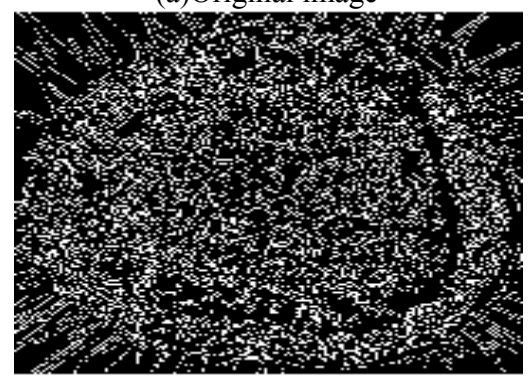

(b) Edge detection

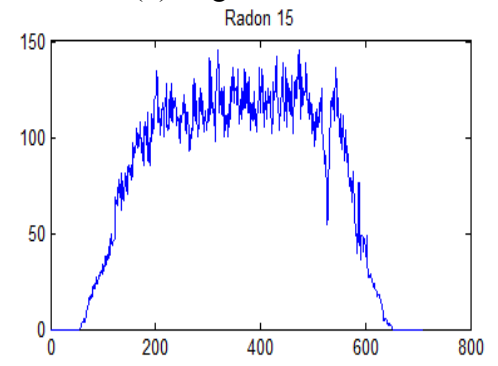

(c) Radon $=15$ 


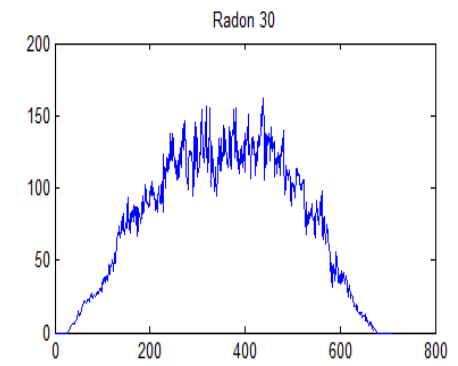

(d) Radon $=30$

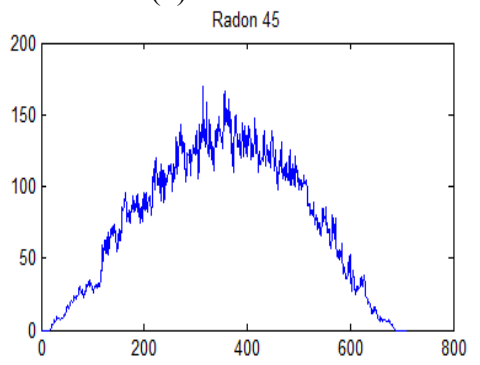

(e) Radon $=45$

Figure 4. Insects 2

As shown in the experiment result, the feature vector extracted by this algorithm basically remains the same when describing the same type of shape and it differ greatly in the description of different shapes. So it has proven that the shape feature extraction method not only has excellent translation, rotation and proportion invariance, but it also has strong shape discrimination ability and it has contained the boundary and texture information of shape. By obtaining the inter- and intra-class discretness, it shows that a shape feature extraction method has a stronger ability to describe shape features. In image recognition and feature extraction, this algorithm can accurately classify and recognize and it has a high recognition rate and it can effectively extract the discrimination features of the image.

\section{CONCLUSION AND FUTURE WORK}

Image invariant features have attracted the attention of many scholars due to their invariance such as scale, angle of view, rotation, illumination and so on. However, in the existing image invariant features, none of them can adapt to all image changes. Each feature has more or less certain limitations, and often only has good invariance for partial transformation, and can only be applied to images of specific scenes. In addition, the invariance of most image invariant features is not very ideal. It is very common that the feature repetition rate is lower than that, and there is still a large space for improvement. This paper based on Radon, studies its relevant theories and image similarity invariant feature extraction based on Radon transform, defines the equation to describe shape regional information through the nature of transform, transforms and normalizes this equation, obtains the similarity invariant feature extraction of multi-angle regional features, and removes the impact of phase on recognition through new similarity computation method. The test experiment proves that the method of this paper is effective and it has more stable rotation, translation, and scale invariance.

The future research work is as follows.

(1) At present, although the method proposed in this paper has high accuracy, the real-time performance is not ideal. It takes too long to calculate the location of potential feature points and screen the real feature points, and the process needs to be optimized.

(2) Because the existing methods can't locate the position of feature points quickly and accurately when calculating the intersection of curves, the discussion on the intersection of curves will be the focus of future work.

\section{ACKNOWLEDGMENT}

This work was supported by the Key Project of Supporting Excellent Youth in Colleges and Universities of Anhui Province (No. gxyqZD2019080), Horizontal Project at Suzhou University of China (No.2020xhx093) and Teaching and Research Project of Anhui Province (2015jxtd041, szxy2020jyxm19).

\section{REFERENCE}

[1] ArinoriInagawa, Kana Saito, Asuka Sasaki, Nobuo Uehara, Dataset for Reproducing Absorption Spectra of Methyl Orange Fromthe RGB Values of Microscopic Images, Data in Brief, 2020, article.105998.

[2] Xie, Qinghua; Zhang, Xiangwei; Cheng, Siyuan. "3D Reconstruction Method of Image Based on Digital Microscope", Acta Microscopica, 2019, vol. 28, no. 5, pp.1289-1300.

[3] Rafik H. Aramyan, Robert M. Mnatsakanov, "To Recovering the Moments From The Spherical Mean Radon Transform", Journal of Mathematical Analysis and Applications, 2020, vol. 490, no. 215, article.124334.

[4] Yali Huang, Zhiwen Liu, Yonggang Shi, Quantitative Analysis of Live Lymphocytes Morphology and Intracellular Motion in Microscopic Images", Biomedical Signal Processing and Control, 2015, vol. 18, no. 4, pp. 195-203.

[5] Benedykt R. Jany, ArkadiuszJanas, Franciszek Krok, "Automatic Microscopic Image Analysis By Moving Window Local Fourier Transform and Machine Learning", Micron, 2020, vol.130, no.3, article. 102800 .

[6] AliGholami, MiladFarshad, Fast Hyperbolic Radon Transform Using Chirp-Z Transform, Digital Signal Processing, 2019, vol. 87, no.4, pp.34-42.

[7] G. Moreno Chávez, Jesús Villa, D. Sarocchi, Efrén González-Ramírez. A Method and Software Solution for Classifying Clast Roundness Based on The Radon Transform", Computers \& Geosciences, 2020, vol.138, no. 5 , article. 104451 .

[8] Navid Alemi Koohbanani, Mostafa Jahanifar, Neda Zamani Tajadin, Nasir Rajpoot, "NuClick: A Deep Learning Framework for Interactive Segmentation of Microscopic Images", Medical Image Analysis, 2020, article.101771.

[9] Longyu Xia, Yue Yao, Yang Dong, Mingzhe Wang, Lan Ma, Mueller Polarimetric Microscopic Images Analysis Based Classification of Breast Cancer Cells, Optics Communications, 2020, vol. 475, 15, article.126194.

[10] Lyu, Shuyi; Wang, Jue. (2019) "Computer Aided Diagnosis of Breast Cancer Based on Ultrasound Image, Investigacion Clinica, vol. 60, no. 3, pp.546-554.

[11] Chai, Guofei; Wang, Hailun; Yang, Mingxia, Multi-Agent Vision Positioning and Coordination Formation Control Strategy of Agricultural Machinery", Revista De La Facultad De Agronomia De La Universidad Del Zulia, 2019, vol. 36, no. 4, pp.942-952. 
[12] Francesca Bartolucci, Filippo De Mari, Ernesto De Vito, Francesca Odone, The Radon Transform Intertwines Wavelets and Shearlets", Applied and Computational Harmonic Analysis, 2019, vol. 47, no. 3, pp.822-847.

[13] Arinorilnagawa, Asuka Sasaki, Nobuo Uehara, Reproducing Absorption Spectra of $\mathrm{Ph}$ Indicators From RGB Values of Microscopic Images", Talanta, 2020, vol. 216, no. 15, article.120952.

[14] Imran Fareed Nizami, Amer Bilal Mann, Khadija Maqbool, Impact of Radon Transform on Plane Wave Diffraction Byan Impedance Step Geometry", Optik, 2018, vol. 175, no. 12, pp.1-7.

[15] Viktor V. Nikitin, Fredrik Andersson, Marcus Carlsson, Anton A. Duchkov, Fast Hyperbolic Radon Transform Represented as Convolutions in Log-Polar Coordinates", Computers \& Geosciences, 2017, vol. 105, no. 8, pp.21-33.
[16] Ambar N. Sengupta, The Gaussian Radon Transform as A Limit of Spherical Transforms", Journal of Functional Analysis, 2016, vol. 271, no. 111 , pp.3242-3268.

\section{Creative Commons Attribution License 4.0 (Attribution 4.0 International, CC BY 4.0)}

This article is published under the terms of the Creative Commons Attribution License 4.0

https://creativecommons.org/licenses/by/4.0/deed.en_US 